\title{
Can Conventional Magnetic Resonance Imaging Substitute Three-Dimensional Magnetic Resonance Imaging in the Diagnosis of Lumbar Foraminal Stenosis?
}

\author{
Maruf Mohammad Hasib ${ }^{1}$, Kentaro Yamada ${ }^{1,2}$, Masatoshi Hoshino ${ }^{1}$, Eiji Yamada ${ }^{3}$, Koji Tamai ${ }^{1}$, \\ Shinji Takahashi ${ }^{1}$, Akinobu Suzuki ${ }^{1}$, Hiromitsu Toyoda ${ }^{1}$, Hidetomi Terai ${ }^{1}$, Hiroaki Nakamura ${ }^{1}$ \\ ${ }^{1}$ Department of Orthopaedic Surgery, Osaka City University, Osaka, Japan \\ ${ }^{2}$ Department of Orthopaedic Surgery, Fuchu Hospital, Osaka, Japan \\ ${ }^{3}$ Division of Central Radiology, Osaka City University, Osaka, Japan
}

Study Design: Retrospective radiological comparative design.

Purpose: To investigate whether conventional magnetic resonance imaging (MRI) could substitute three-dimensional (3D)-MRI for the calculation of the foraminal stenotic ratio (FSR) and clarification of which patients can be assessed more accurately using 3D-MRI. Overview of Literature: Previous studies have indicated that 3D-MRI is useful for diagnosing lumbar foraminal stenosis. The FSR obtained using 3D-MRI, described as the ratio of stenosis length, characterized by perineural fat obliteration, to the length of the entire foramen, could indicate the stenosis severity; however, this method is time-consuming and expensive. The FSR also can be calculated using conventional MRI.

Methods: We investigated 154 foramina at L5-S1 in 77 patients. All the patients had degenerative lumbar disorders and had undergone both conventional MRI and 3D-MRI during the same visit. Differences between the FSRs calculated from conventional and 3DMRI reconstructions and any correlations with the plain radiography findings were assessed.

Results: In foramina that had a FSR of $<50 \%$ on conventional MRI, the difference between the FSR obtained using conventional MRI and 3D-MRI was $5.1 \%$, with a correlation coefficient of 0.777 . For foramina with a FSR $\geq 50 \%$ on conventional MRI, the difference was $20.2 \%$, with a correlation coefficient of 0.54 . FSR obtained using 3D-MRI was significantly greater in patients who required surgery than in those who were successfully treated with conservative methods ( $88 \%$ and $42 \%$, respectively). Segments with spondylolisthesis or lateral wedging showed higher FSRs than those without these conditions on both types of MRI.

Conclusions: FSRs $<50 \%$ obtained using conventional MRI were sufficiently reliable; however, the results were inaccurate for FSRs $\geq 50 \%$. Patients with high FSRs on 3D-MRI were more likely to require surgical treatment. Therefore, 3D-MRI is recommended in patients with suspected stenosis detected using conventional MRI or plain radiographs.

Keywords: Lumbar foraminal stenosis; Three-dimensional magnetic resonance imaging; Perineural fat obliteration; Surgery; Conservative treatment

Received Mar 29, 2020; Revised Apr 18, 2020; Accepted May 12, 2020

Corresponding author: Kentaro Yamada

Department of Orthopaedic Surgery, Osaka City University Graduate School of Medicine, 1-4-3 Asahi-machi, Abeno-ku, Osaka 5458585, Japan

Tel: +81-6-6645-3851, Fax: +81-6-6646-6260, E-mail: yamachen@msic.med.osaka-cu.ac.jp 


\section{Introduction}

Lumbar foraminal stenosis (LFS) is a relatively common cause of lumbar radiculopathy and is attributable to the narrowing of the nerve root foramen, with a reported incidence rate of $8 \%-26 \%$ [1-4]. L5 is most commonly involved (75\%) [3,5,6]. Osteophytes, ligamentous hypertrophy, or disc degeneration can cause LFS in the anteroposterior and/or craniocaudal directions [3]. The L5 transverse process, sacral ala, or lumbosacral ligament may cause stenosis in the L5-S1 extraforaminal zone $[7,8]$. Inaccurate preoperative diagnosis of LFS may cause failed back surgery syndrome [9].

Obliteration of the perineural fat surrounding the nerve root on parasagittal images on magnetic resonance imaging (MRI) is the established marker of LFS $[10,11]$. As per some reports, conventional MRI is inaccurate in certain cases $[2,4]$. Many techniques have been proposed for the evaluation of LFS, such as oblique views on three-dimensional MRI (3D-MRI) [12-15], MR myelography [4,16] or radiculography [17], diffusion-weighted imaging [18], and electrophysiological methods [19]. Among them, 3DMRI sequences are obtained from thin-section thickness images. Therefore, 3D-MRI sequence can be produced with direct visualization of the nerve root on 3D rendering images or oblique reconstructed image in any plane. The foraminal stenotic ratio (FSR) using reconstructed oblique images with 3D-MRI (Fig. 1) is reportedly an accurate method for assessing the stenosis severity to identify patients who need surgical treatment [15].

The FSR is calculated as per the degree of fat obliteration in the neural foramen. However, FSR measurement
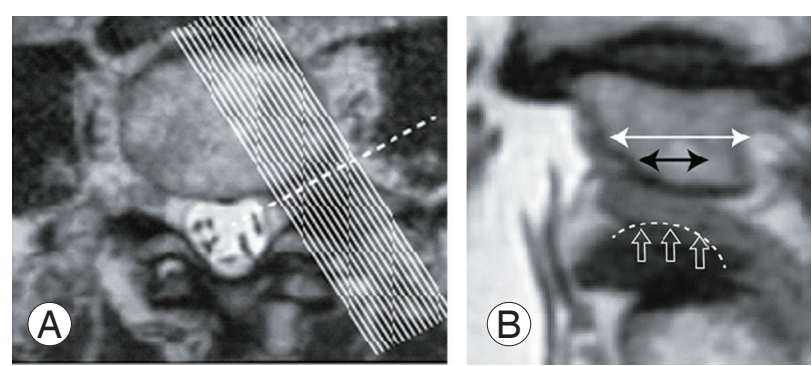

Fig. 1. Evaluation of foraminal stenotic ratio (FSR). (A) FSR was defined as the degree of stenosis in the neural foramen. (B) The oblique coronal image by three-dimensional magnetic resonance imaging showed foraminal stenosis at medial side of the foramen due to disc bulging in this case (dot line and box arrow). FSR were evaluated as the ratio of length of stenosis (black arrow) to length of the foramen (white arrow). FSR=length of stenosis/length of fora$\operatorname{men} \times 100 \%$. with 3D-MRI is time-consuming; moreover, additional time and cost are needed to perform 3D-MRI in addition to conventional MRI. FSR can be calculated even using conventional MRI. In this study, we compared the FSR using both, conventional and 3D-MRIs with plain radiographs in patients who had lumbar degenerative disease. This study was designed to determine whether conventional MRI can substitute 3D-MRI in the measurement of FSR and to identify patients in whom 3D-MRI can be effectively used.

\section{Materials and Methods}

We collected MRI images obtained using conventional MRI and 3D-MRI during the same visit and performed retrospective evaluation of the outpatient data from one institution. Patients aged $\geq 40$ years who had visited outpatient clinics for lumbar degenerative disease both preoperatively and postoperatively from Feb 2017 to August 2018 and for whom conventional as well as 3D-MRI images were obtained during the same visit were enrolled. Those with duplicate MRIs, prior surgery at the L5-S1 or instrumentation at L5, spinal infection, acute trauma, spinal malignant tumor, or congenital deformities were excluded. This study protocol was approved by the Institutional Review Board of Osaka City University (approval no., 3807; approval date: August 3, 2017). Our study assessed patient acceptability of opt-out consent for secondary use of patient data.

LFS was diagnosed based on clinical symptoms supporting selective nerve root block and MRI findings of perineural fat obliteration or nerve root compression in the lumbar foramen on the parasagittal images of conventional MRI or non-reconstructed coronal images of 3DMRI. LFS treatment was standardized as follows: First, conservative treatment was provided, including oral analgesia, physiotherapy, epidural steroids, or selective nerve blocks. Surgery was performed only for patients who were unresponsive to conservative treatment for at least 3 month or who exhibited severe motor weakness.

A total of 77 patients underwent both conventional and 3D-MRI examination at the same visit during the investigation period and fulfilled the inclusion criteria. We investigated their 154 foramina at the L5-S1 in this study. Among these, 10 patients with 11 affected foramina underwent surgical treatment, and six patients with six foramina at the L5-S1 underwent conservative treatment. 
The asymptomatic side of the foramen in symptomatic patients took into asymptomatic foramina. There were 80 asymptomatic foramina. In 33 cases with 57 foramina, we were unable to determine the level responsible for L5 radiculopathy because of L4-5 intra-canal stenosis. We investigated the difference between FSRs at L5-S1, as measured on conventional and 3D-MRI and with plain radiography.

\section{Magnetic resonance imaging settings}

All the MRI examinations were performed using a 3.0 T MR system (Philips Achieva; Philips, Amsterdam, The Netherlands), using a spine matrix coil provided by the manufacturer. 3D-MRI sequence and conventional MRI images were obtained during the same visit with a 20-minute examination frame. The 3D-MRI sequences were coronal based 3D-T2-weighted sampling perfection with application optimized contrast using different flip angle evolution (SPACE). The scanner settings were as follows: repetition time (TR), 1,700 ms; echo time (TE), $110 \mathrm{~ms}$; slice thickness, $0.70 \mathrm{~mm}$; field of view, $300 \mathrm{~mm}$; matrix, 352×253; and imaging times, 5 minutes 7 seconds. The conventional MRI sequences were sagittal T1-weighted images (T1WI): TR, $500 \mathrm{~ms}$; TE, 9 ms; slice thickness, $4 \mathrm{~mm}$; field of view, $280 \mathrm{~mm}$; and matrix, 352×246.

For 3D-MRI evaluation, multiplanar images in an oblique plane were reconstructed using workstation software (Synapse Vincent; Fujifilm Corp., Tokyo, Japan). The oblique images were reconstructed as per a previously reported method [15]: reconstruction of planes from the line of the lower endplate of the L5 vertebral body, with the planes parallel to the L5-S1 foramen on the axial plane. The oblique coronal image was chosen by the slice that showed the L5 lumbar nerve root from the medial to the lateral portions of the foramen. Oblique sagittal images were reconstructed as the vertical plane to the oblique coronal images with a $1.0-\mathrm{mm}$ gap. We reviewed all the oblique sagittal slices across the L5-S1 foramen that included the extraforaminal zone (Fig. 2).

\section{Parameters from magnetic resonance imaging and plain radiographs}

The FSR was evaluated on both the conventional and 3DMRI images as per a previous study [15]. Stenosis was defined as perineural fat obliteration on the parasagittal images using conventional MRI (Fig. 3) or on oblique sagittal images using 3D-MRI (Fig. 4). FSR was calculated as the ratio of the slice numbers showing stenosis to the slice numbers including the entire foramen, both

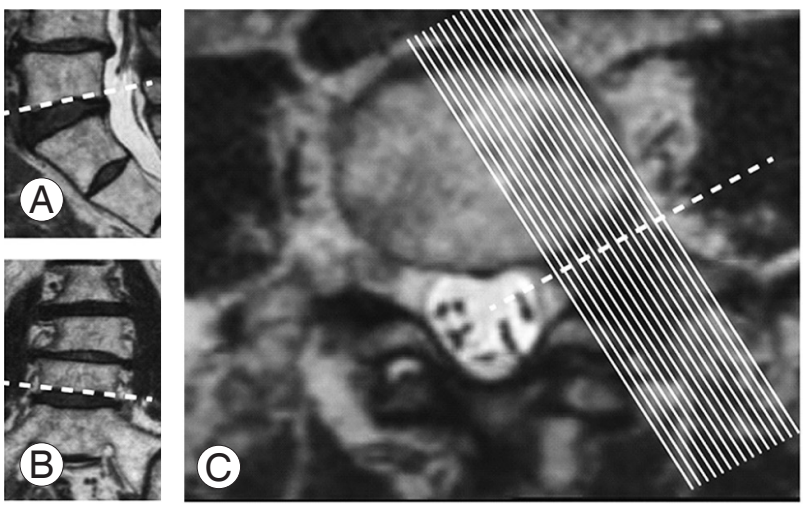

Fig. 2. (A-C) Reconstruction of images for evaluation of foraminal stenotic ratio (FSR) by three-dimensional magnetic resonance imaging. The oblique plane was adjusted to the L5 lower end plate (sagittal and coronal plane) and the L5S1 foramen (axial plane). FSR were evaluated by all oblique sagittal images on neural foramen across the $L 5$ pedicle.
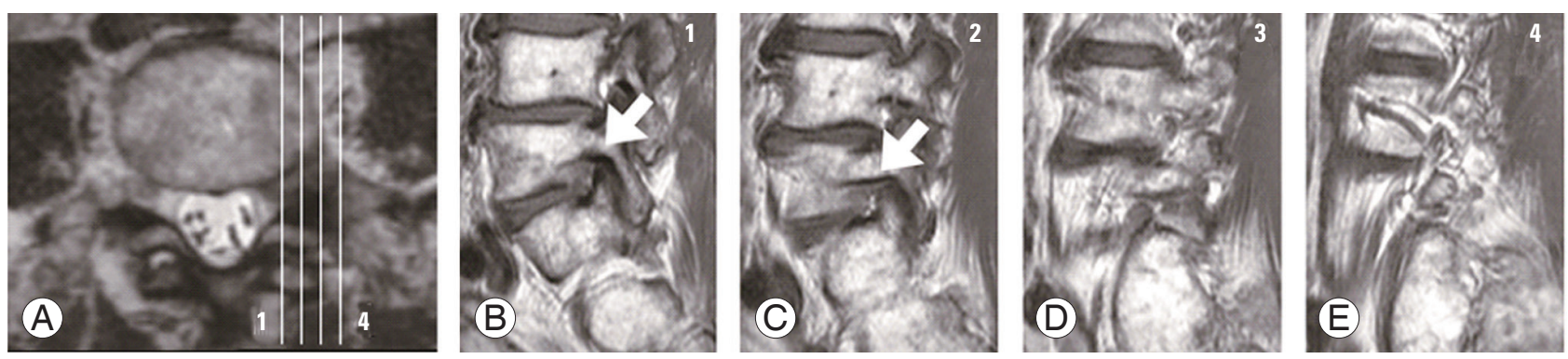

Fig. 3. Foraminal stenotic ratio (FSR) by conventional magnetic resonance imaging. (A-E) Parasagittal images of neural foramen were evaluated. Slices with obliteration of perineural fat (white arrow) were regarded as stenosis positive. FSR was calculated by a ratio of slice numbers of stenosis positive to the slice numbers of foramen. FSR of this case were 2 over 4, 50\%. 

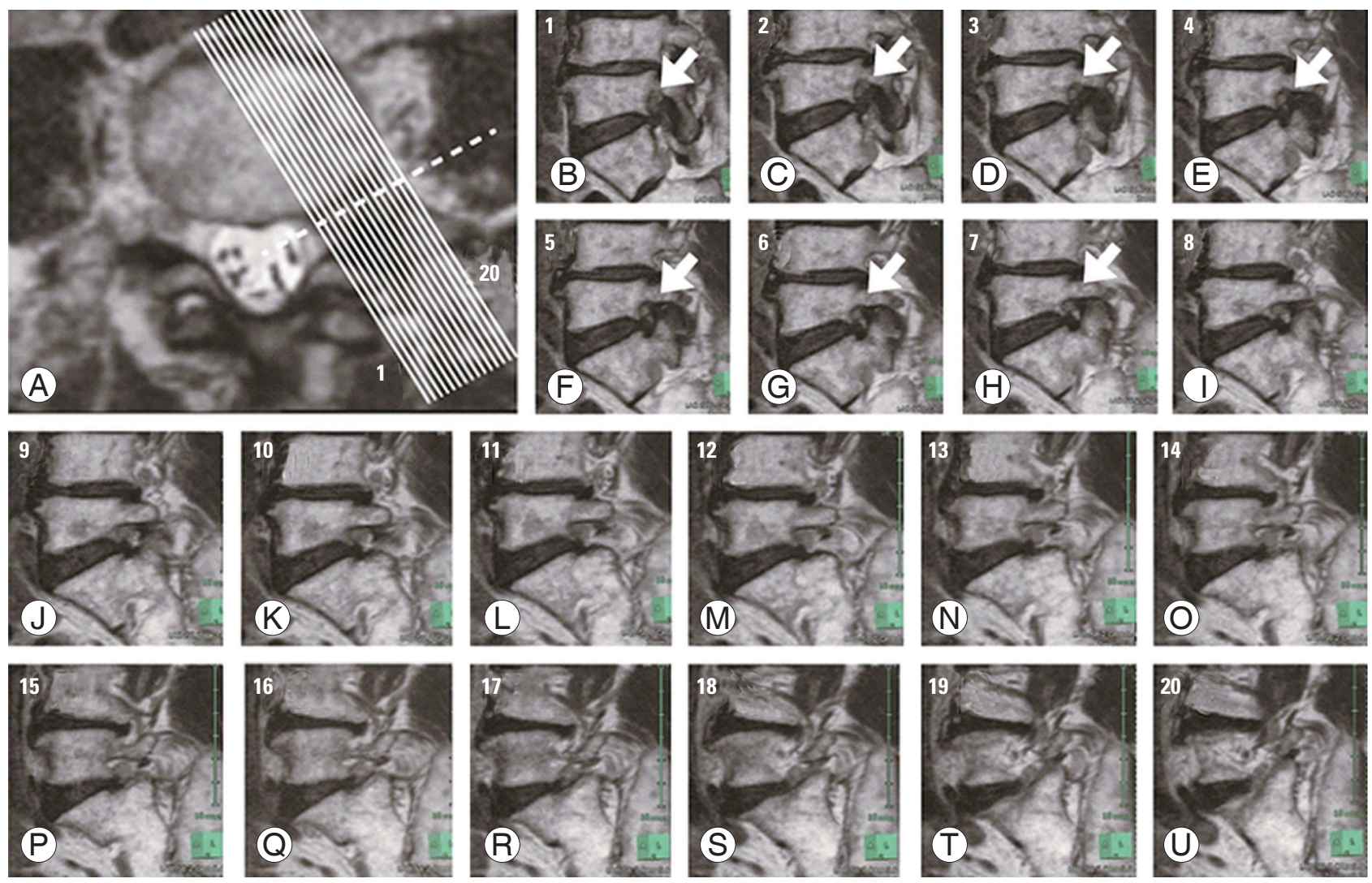

Fig. 4. Foraminal stenotic ratio (FSR) by three-dimensional magnetic resonance imaging. (A-U) Oblique sagittal images of neural foramen were evaluated. Slices with obliteration of perineural fat (white arrow) were regarded as stenosis positive. FSR was calculated by a ratio of slice numbers of stenosis positive to the slice numbers of foramen. FSR of this case were 7 over $20,35 \%$.

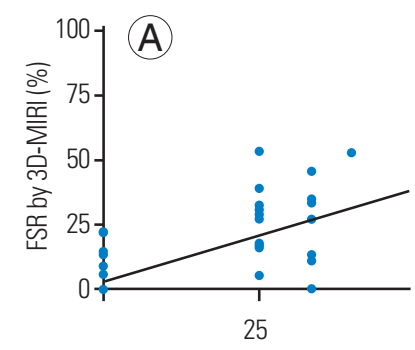

FSR by conventional MRI (\%)

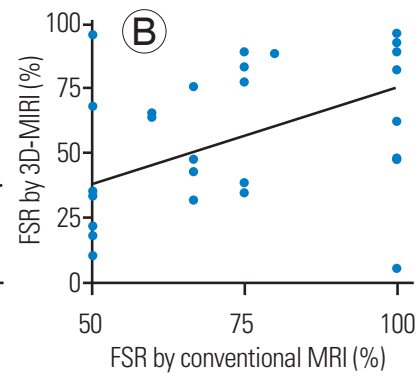

Fig. 5. Scatter plot of foraminal stenotic ratio (FSR) by conventional and threedimensional magnetic resonance imaging (3D-MRI). (A) Foramina with FSR by conventional $\mathrm{MRI}<50 \%$. The difference of absolute value was $5.1 \%$ and correlation coefficient was $0.777(p<0.001)$. (B) Foramina with FSR by conventional MRI $\geq 50 \%$. The difference of absolute value was $20.2 \%$ and correlation coefficient was $0.54(p<0.001)$.

on the conventional MRI (Fig. 3) and 3D-MRI (Fig. 4). In order to confirm measurement reliability, FSR was calculated in ten randomly chosen foramina using conventional MRI and 3D-MRI twice by one author (K.Y.) at a 3-month interval and by two authors (K.Y. and M.M.H.) independently who were blinded to the clinical informa- tion of the patients. Before measurement, the authors K.Y. and M.M.H. evaluated five other patients together to understand the measurement methods. The intra-class and inter-class correlation coefficients for the parameters were as follows: FSR using conventional MRI: 0.883 (95\% confidence interval [CI], 0.733-0.952) and 0.883 (95\% CI, 0.732-0.952), FSR using 3D-MRI: 0.958 (95\% CI, $0.9-0.984)$ and 0.89 (95\% CI, 0.746-0.955), respectively.

Plain standing lumbar radiographs were compared using MRI examination. We evaluated spondylolisthesis and lateral wedging. Anterior spondylolisthesis and posterior retrolisthesis were defined as positive if they measured $\geq 3 \mathrm{~mm}$ slip of L5 to S1 on the lateral radiographs. Lateral wedging of a vertebral segment was defined $\geq 2^{\circ}$ on the anteroposterior radiograph.

\section{Statistical analyses}

Results are represented as mean \pm standard deviation values. Differences between the FSRs obtained using con- 
Table 1. Differences of FSR by conventional and 3D-MRI among patients regarding lumbar foraminal stenosis at L5-S1

\begin{tabular}{|c|c|c|c|c|}
\hline Variable & Surgery $(\mathrm{N}=11)$ & Conservative ( $\mathrm{N}=6)$ & Asymptomatic ( $\mathrm{N}=80)$ & $p$-value \\
\hline FSR by conventional MRI (\%) & $76.4 \pm 26.2^{\text {a) }}$ & $54.2 \pm 18.8^{\mathrm{a})}$ & $17.6 \pm 3.2$ & $<0.0001$ \\
\hline FSR by 3D-MRI (\%) & $87.7 \pm 15.1^{\mathrm{al}, \mathrm{bl}}$ & $41.7 \pm 19.6^{a)}$ & $16.3 \pm 22.9$ & $<0.0001$ \\
\hline
\end{tabular}

Values are presented as mean \pm standard deviation. Patients divided into surgery: requiring surgical treatment for lumbar foraminal stenosis at L5-S, successful conservative treatment for lumbar foraminal stenosis at L5-S1, and asymptomatic of $\mathrm{L} 5$ radiculopathy. All $p$-values were analyzed by Kruskal-Wallis test.

FSR, foraminal stenotic ratio; 3D-MRI, three-dimensional magnetic resonance imaging.

a) $p<0.05$ compared with the asymptomatic group. ${ }^{\text {b) }} p<0.05$ compared with the conservative group.

Table 2. Association between findings of plain radiograph and 3D-MRI

\begin{tabular}{|c|c|c|c|c|c|c|c|}
\hline \multirow{2}{*}{ Variable } & \multicolumn{4}{|c|}{ Association between spondylolisthesis and FSR } & \multicolumn{3}{|c|}{$\begin{array}{l}\text { Association between sides and FSR } \\
\text { in case with lateral wedging }\end{array}$} \\
\hline & $\begin{array}{l}\text { Anterior spondylolisthesis } \\
\qquad(\mathrm{N}=16)\end{array}$ & $\begin{array}{l}\text { Retrolisthesis } \\
\qquad(\mathrm{N}=16)\end{array}$ & $\begin{array}{l}\text { Spondylolisthesis (-) } \\
\qquad(\mathrm{N}=118)\end{array}$ & $p$-value ${ }^{\text {a) }}$ & $\begin{array}{l}\text { Concave } \\
\text { side }\end{array}$ & $\begin{array}{l}\text { Convex } \\
\text { side }\end{array}$ & $p$-value \\
\hline FSR by conventional MRI (\%) & $54.3 \pm 32.9^{b)}$ & $33 \pm 28.9$ & $21.0 \pm 30.9$ & $<0.001$ & $45.6 \pm 5$ & $15.0 \pm 21.3$ & $<0.001$ \\
\hline FSR by 3D-MRI (\%) & $46.0 \pm 33^{b)}$ & $40.7 \pm 31.9^{b)}$ & $18.3 \pm 26.7$ & $<0.001$ & $44.4 \pm 4.9$ & $13.4 \pm 3.0$ & $<0.001$ \\
\hline
\end{tabular}

Values are presented as mean \pm standard deviation. Spondylolisthesis was defined as $\geq 3 \mathrm{~mm}$ slip on standing lateral radiographs; lateral wedging was defined as $\geq 2^{\circ}$ lateral wedging on standing anteroposterior radiographs.

3D-MRI, three-dimensional magnetic resonance imaging; FSR, foraminal stenotic ratio.

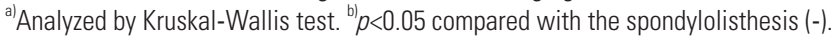

ventional and 3D-MRI were examined with Spearman's rank correlation coefficient in all 154 L5-S1 foramina. The Mann-Whitney $U$-test was performed for continuous variables, and the chi-squared test was used for categorical variables between the two groups. The Kruskal-Wallis test and a post hoc analysis using Turkey's test were performed to compare the differences in symptom severity. A $p$-value $<0.05$ was considered statistically significant. All the statistical analyses were performed using IBM SPSS ver. 19.0 (IBM Corp., Armonk, NY, USA).

\section{Results}

\section{Differences in foraminal stenotic ratio measurements between conventional and three-dimensional mag- netic resonance imaging}

The evaluated number of slices for a foramen was significantly larger with 3D-MRI than with conventional MRI $(20.2 \pm 2.4$ and $3.8 \pm 0.7$, respectively; $p<0.001)$. As a whole, the difference in the absolute value between FSR obtained using conventional and that obtained using 3D-MRI was $9.6 \%$, and the correlation coefficient was $0.893(p<0.001)$. In the foramina with FSR $<50 \%$ on conventional MRI, the difference was $5.1 \%$, and the correlation coefficient was
0.777 (Fig. 5A). In contrast, the difference was $20.2 \%$ and the correlation coefficient was 0.540 in the foramina with FSR $\geq 50 \%$ on conventional MRI (Fig. $5 B$ ).

Subsequently, we investigated each FSR using conventional and 3D-MRI among patients who required surgical treatment for LFS at L5-S1, those with successful conservative treatment for LFS at L5-S1, and those without symptoms of L5 radiculopathy. For FSRs obtained using both, conventional and 3D-MRI, there were significant differences between patients who required surgery and those who had no symptoms $(p<0.001$ and $p<0.001$, respectively) and between patients with successful conservative treatment and those without symptoms ( $p=0.007$ and 0.021 , respectively). However, the FSR obtained using 3D-MRI showed significant differences between patients with successful conservative treatment and those who required surgery ( $41.7 \%$ and $87.7 \%, p<0.001)$; however, the FSR obtained using conventional MRI did not show any such differences (Table 1).

\section{Association between findings on plain radiographs and three-dimensional magnetic resonance imaging}

There were eight patients (10.4\%) with anterior spondylolisthesis, 8 (10.4\%) with retrolisthesis, and 46 (59.7\%) 
Table 3. Relationship between findings of plain radiograph and clinical symptom

\begin{tabular}{lccc} 
Variable & $\begin{array}{c}\text { Symptomatic } \\
\text { foraminal stenosis } \\
\text { at L5-S1 }\end{array}$ & $\begin{array}{c}\text { Asymptomatic for } \\
\text { foraminal } \\
\text { stenosis at L5-S1 }\end{array}$ & p-value \\
\hline Spondylolisthesis of L5 & $3(21.4)$ & $2(7.1)$ & 0.332 \\
\hline Anterior & $1(7.1)$ & $1(3.6)$ & \\
\hline Posterior & $10(71.4)$ & $25(89.3)$ & \\
\hline Spondylolisthesis (-) & & & 0.092 \\
\hline Lateral wedging at L5-S1 & $14(77.8)$ & $18(64.3)$ & \\
\hline Wedging (+) & $2(12.5)$ & $10(35.7)$ & \\
\hline Wedging (-) & & & \\
\hline
\end{tabular}

Values are presented as number (\%).

with lateral intervertebral disc wedging at L5-S1 on plain standing radiographs. Patients with spondylolisthesis showed significantly higher FSRs than those without spondylolisthesis on both, conventional and 3D-MRI. Patients with retrolisthesis showed significantly higher FSR on 3D-MRI than those without spondylolisthesis (Table 2). The FSRs of the concave sides were significantly higher than those of the convex sides in patients with lateral wedging. However, there was no significant difference between patients with symptomatic foraminal stenosis at L5-S1 and those without symptoms for L5 radiculopathy in either spondylolisthesis or the side of lateral disc wedging (Table 3).

\section{Representative case}

Fig. 6 shows an 82-year-old male who experienced left leg pain. MRI showed no intra-canal stenosis at L4-5 and nerve root entrapment in the medial zone of the left L5S1 foramen. Plain radiographs show lateral wedging of $2^{\circ}$ with concavity on the left side. FSR using conventional MRI is shown in Fig. 3. FSR with 3D-MRI is shown in Fig. 4. There was discrepancy between FSR obtained using conventional MRI (50\%) and 3D-MRI (35\%). This patient has been successfully treated conservatively for 1.5 years.

\section{Discussion}

The diagnosis of LFS remains challenging, and several diagnostic tools have been reported. FSR using 3D-MRI is a useful diagnostic parameter [15]; a larger FSR indicates more extensive foraminal stenosis. However, 3D-MRI is
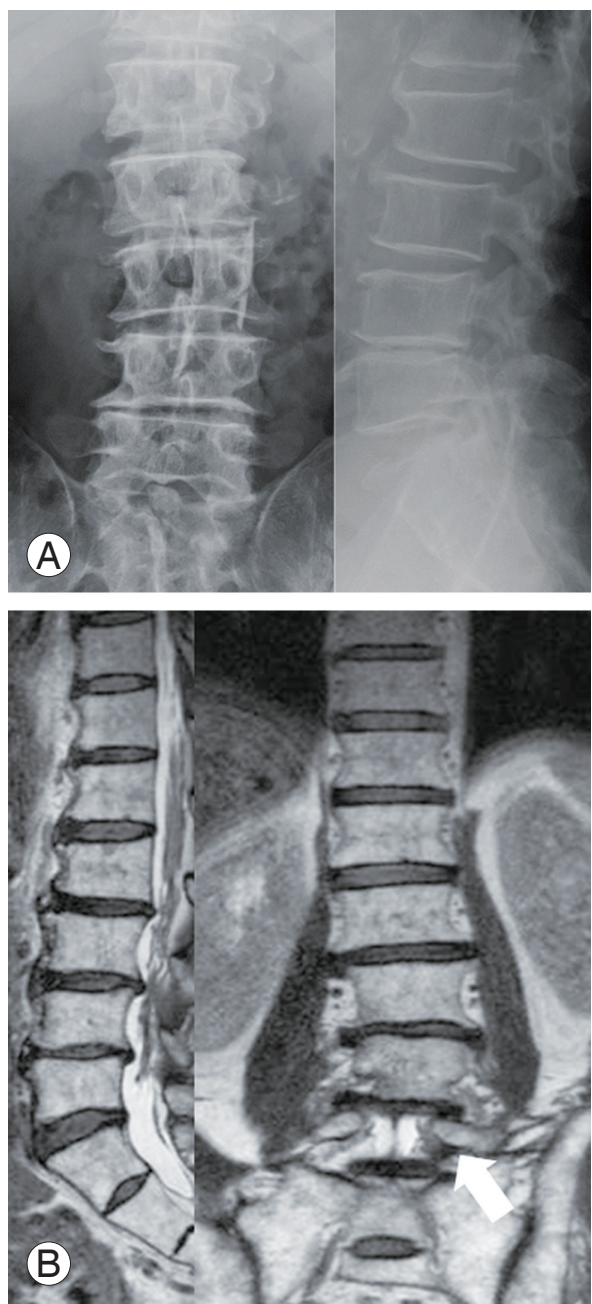

Fig. 6. Representative case of an 82-year-old male who has succeeded conservative treatment for 1.5 year. (A) Plain radiograph. Lateral wedging of $2^{\circ}$ with concave of left side was observed. (B) Conventional magnetic resonance imaging. There was no intra-canal stenosis at $L 4-5$ and nerve root entrapment in the medial zones of the left L5-S1 foramen (white arrow).

not routinely performed for patients suspected to have lumbar degenerative disorders, with increasing medical costs becoming an issue. It is necessary to clarify the requirements for 3D-MRI to acquire detailed information for LFS to judge the indication for surgical intervention. Therefore, it is important to identify features related to a higher FSR in routine radiological examinations. The present results showed that FSR obtained using conventional MRI had a sufficiently strong correlation $(r=0.777)$ with FSR obtained using 3D-MRI in cases with no/slight stenosis $(<50 \%)$; however, this correlation was not strong $(r=0.540)$ in cases with stenosis $\geq 50 \%$. This result suggests that moderate/large FSRs obtained using conventional MRI did not always correlate with moderate/large FSRs 
obtained using 3D-MRI. FSR also correlated with the findings on plain radiographs, such as anterior/posterior spondylolisthesis and lateral wedging. To our knowledge, this is the first study to identify patients who should undergo 3D-MRI as per the FSR calculation.

The FSR assesses perineural fat obliteration in the entire neural foramen. Perineural fat obliteration on parasagittal MRI has previously been reported as the most suggestive indicator of stenosis $[3,10,11]$. However, conventional fat obliteration evaluation methods described in the classification of Lee et al. [11] have certain limitations in evaluating stenosis severity. One flaw arises from the evaluation of only one slice in the lumbar foramen. The information from one slice limits the evaluation and might give falsepositive or false-negative results $[2,4,15]$. We attempted to overcome this flaw by evaluating all slices in the neural foramen to generate the FSR. By comparing patients who require surgery and those for whom conservative treatment was successful in our previous report of FSR using 3D-MRI, symptomatic patients with $\geq 50 \%$ FSR required surgical treatment with a positive predictive value of $70.6 \%[15]$.

In this study, there was a discrepancy between FSR obtained using conventional MRI and that obtained using 3D-MRI, especially in cases with $\geq 50 \%$ FSR on conventional MRI. There are two possible causes; one is the difference in slice thickness for evaluation. The 3D-MRI evaluation included 13-25 slices in contrast with the conventional MRI evaluation that involved 2-5 slices. Information that could be present in the slices that were not evaluated is important for determining the stenosis severity. Second, FSR obtained using conventional MRI evaluated parasagittal images, while the FSR obtained using 3D-MRI evaluated oblique images. Parasagittal images alone are difficult to evaluate for stenosis in the extraforaminal zone $[17,20]$ or for patients with spinal deformities, such as scoliosis and severe lordosis $[2,4,21]$. Oblique images can describe the entire nerve root pathway in the foramen, including the extraforaminal zone $[12,13,20,22]$. Oblique sagittal images allow circumferential evaluation in both the craniocaudal and antero-posterior directions in the entire pathway of the foramen. Moreover, reconstruction from 3D-MRI images could easily provide images parallel to the disc and foramen, even in patients with spinal deformity. Therefore, detailed evaluation of LFS with conventional MRI was invalid in cases with suspected LFS, even when using the FSR technique. This was supported by the present findings. The FSR obtained using 3D-MRI showed a significant difference between patients who were successfully managed with conservative treatment and those who required surgery ( $42 \%$ and $88 \%$ ), in agreement with a previous report [15]; however, the FSR obtained using conventional MRI did not show any such difference. Therefore, patients with FSR $\geq 50 \%$ obtained using conventional MRI are recommended to undergo additional evaluation with 3D-MRI for evaluation of the stenosis severity.

The relationship between findings on plain radiographs/ computed tomography and LFS have been addressed in some reports [23-25]. They indicated that spondylolisthesis with dynamic instability or on the concave side of a scoliosis were related to foraminal stenosis. Our results were consistent with their results; the level of anterior/ posterior spondylolisthesis or concave side of the lateral disc wedging level showed larger FSRs. However, symptomatic LFS was not related to the findings on plain radiographs. Therefore, additional examination using 3D-MRI is not recommended for patients with only findings on plain radiographs.

The 3D-MRI with coronal based T2 SPACE sequence and the conventional sagittal T1WI in this study protocol could be obtained within one examination frame of MRI. Owing to reconstruct to sagittal or axial images from coronal images of T2 SPACE sequence, we could diagnose degenerative disorders and discriminate from fracture, infection, or spinal tumor sufficiently without increased burden to the patients. Therefore, the MRI protocol of this study might be recommended for patients with findings on plain radiographs and clinical symptoms of radiculopathy instead of routine MRI.

The present study has certain limitations. First, the MRI sequences differed between conventional MRI and 3DMRI; conventional MRI used T1WIs and the 3D-MRI used T2-weighted images (T2WIs). Although the reason was to maximize accuracy for the diagnosis of various pathologies, the ideal study protocol to compare the FSRs obtained using conventional MRI and 3D-MRI should employ either T1WI or T2WI. There is no consensus on which sequence is better for the evaluation of the lumbar foramina; previous reports have recommended T1WI $[3,11]$. In contrast, a more recent study has reported good diagnostic results using T2WIs [22]. In terms of evaluating perineural fat obliteration, fat is hyperintense in both T1WIs and T2WIs. Therefore, the measurement error 
caused by varied MRI sequences did not cause a major problem. Second, the sample size was relatively small, and consecutive patients were not enrolled. However, we believe that the present findings are valuable to emphasize the importance of reducing the healthcare cost. Future prospective studies on a larger sample study are warranted to confirm the discrepancy between conventional MRI and 3D-MRI.

\section{Conclusions}

Conventional MRI cannot fully substitute 3D-MRI for FSR evaluation. The FSRs obtained using conventional MRI were sufficiently reliable for patients with $<50 \%$ stenosis; however, the reliability was weak in patients with $\geq 50 \%$ stenosis. FSR was related to the levels of anterior/ posterior spondylolisthesis or the concave side in patients with lateral disc wedging. Patients with high FSR on 3DMRI were likely to require surgical treatment. Therefore, 3D-MRI is recommended only in symptomatic cases with suspected stenosis on conventional MRI or plain radiographs.

\section{Conflict of Interest}

No potential conflict of interest relevant to this article was reported.

\section{Author Contributions}

Mohammad Hasib Maruf wrote and prepared the manuscript. Kentaro Yamada built conception and design, analyzed the data for the work, and critically revised the article. All authors have read, reviewed, and approved the article.

\section{References}

1. Porter RW, Hibbert C, Evans C. The natural history of root entrapment syndrome. Spine (Phila Pa 1976) 1984;9:418-21.

2. Kunogi J, Hasue M. Diagnosis and operative treatment of intraforaminal and extraforaminal nerve root compression. Spine (Phila Pa 1976) 1991;16:1312-20.

3. Jenis LG, An HS. Spine update. Lumbar foraminal stenosis. Spine (Phila Pa 1976) 2000;25:389-94.

4. Aota Y, Niwa T, Yoshikawa K, Fujiwara A, Asada T,
Saito T. Magnetic resonance imaging and magnetic resonance myelography in the presurgical diagnosis of lumbar foraminal stenosis. Spine (Phila Pa 1976) 2007;32:896-903.

5. Yamada K, Matsuda H, Nabeta M, Habunaga H, Suzuki A, Nakamura H. Clinical outcomes of microscopic decompression for degenerative lumbar foraminal stenosis: a comparison between patients with and without degenerative lumbar scoliosis. Eur Spine J 2011;20:947-53.

6. Yamada K, Matsuda H, Cho H, Habunaga H, Kono $\mathrm{H}$, Nakamura $\mathrm{H}$. Clinical and radiological outcomes of microscopic partial pediculectomy for degenerative lumbar foraminal stenosis. Spine (Phila Pa 1976) 2013;38:E723-31.

7. Nathan H, Weizenbluth M, Halperin N. The lumbosacral ligament (LSL), with special emphasis on the "lumbosacral tunnel" and the entrapment of the 5th lumbar nerve. Int Orthop 1982;6:197-202.

8. Wiltse LL, Guyer RD, Spencer CW, Glenn WV, Porter IS. Alar transverse process impingement of the L5 spinal nerve: the far-out syndrome. Spine (Phila $\mathrm{Pa}$ 1976) 1984;9:31-41.

9. Burton CV, Kirkaldy-Willis WH, Yong-Hing K, Heithoff KB. Causes of failure of surgery on the lumbar spine. Clin Orthop Relat Res 1981:191-9.

10. Wildermuth S, Zanetti M, Duewell S, et al. Lumbar spine: quantitative and qualitative assessment of positional (upright flexion and extension) MR imaging and myelography. Radiology 1998;207:391-8.

11. Lee S, Lee JW, Yeom JS, et al. A practical MRI grading system for lumbar foraminal stenosis. AJR Am J Roentgenol 2010;194:1095-8.

12. Heo DH, Lee MS, Sheen SH, Cho SM, Cho YJ, Oh SM. Simple oblique lumbar magnetic resonance imaging technique and its diagnostic value for extraforaminal disc herniation. Spine (Phila Pa 1976) 2009;34:2419-23.

13. Nemoto O, Fujikawa A, Tachibana A. Three-dimensional fast imaging employing steady-state acquisition MRI and its diagnostic value for lumbar foraminal stenosis. Eur J Orthop Surg Traumatol 2014;24 Suppl 1:S209-14.

14. Yamada $H$, Terada $M$, Iwasaki $H$, et al. Improved accuracy of diagnosis of lumbar intra and/or extraforaminal stenosis by use of three-dimensional MR imaging: comparison with conventional MR imaging. 
J Orthop Sci 2015;20:287-94.

15. Yamada K, Abe Y, Satoh S, Yanagibashi Y, Hyakumachi T, Masuda T. A novel diagnostic parameter, foraminal stenotic ratio using three-dimensional magnetic resonance imaging, as a discriminator for surgery in symptomatic lumbar foraminal stenosis. Spine J 2017;17:1074-81.

16. Kojima A, Torii Y, Morioka S, Sasao Y. Quantification of L5 radiculopathy due to foraminal stenosis using three-dimensional magnetic resonance myelography. Spine Surg Relat Res 2017;1:146-51.

17. Byun WM, Jang HW, Kim SW. Three-dimensional magnetic resonance rendering imaging of lumbosacral radiculography in the diagnosis of symptomatic extraforaminal disc herniation with or without foraminal extension. Spine (Phila Pa 1976) 2012;37:840-4.

18. Eguchi Y, Kanamoto H, Oikawa Y, et al. Recent advances in magnetic resonance neuroimaging of lumbar nerve to clinical applications: a review of clinical studies utilizing Diffusion Tensor Imaging and Diffusion-weighted magnetic resonance neurography. Spine Surg Relat Res 2017;1:61-71.

19. Ando M, Tamaki T, Kawakami M, et al. Electrophysiological diagnosis using sensory nerve action potential for the intraforaminal and extraforaminal L5 nerve root entrapment. Eur Spine J 2013;22:833-9.
20. Takeuchi M, Wakao N, Kamiya M, et al. Lumbar extraforaminal entrapment: performance characteristics of detecting the foraminal spinal angle using oblique coronal MRI: a multicenter study. Spine J 2015;15:895-900.

21. Lee CK, Rauschning W, Glenn W. Lateral lumbar spinal canal stenosis: classification, pathologic anatomy and surgical decompression. Spine (Phila Pa 1976) 1988;13:313-20.

22. Lee S, Jee WH, Jung JY, Lee SY, Ryu KS, Ha KY. MRI of the lumbar spine: comparison of 3D isotropic turbo spin-echo SPACE sequence versus conventional 2D sequences at 3.0 T. Acta Radiol 2015;56:174-81.

23. Ploumis A, Transfeldt EE, Gilbert TJ Jr, Mehbod AA, Dykes DC, Perra JE. Degenerative lumbar scoliosis: radiographic correlation of lateral rotatory olisthesis with neural canal dimensions. Spine (Phila Pa 1976) 2006;31:2353-8.

24. Kaneko Y, Matsumoto M, Takaishi H, Nishiwaki Y, Momoshima S, Toyama Y. Morphometric analysis of the lumbar intervertebral foramen in patients with degenerative lumbar scoliosis by multidetector-row computed tomography. Eur Spine J 2012;21:2594602.

25. Yamada K, Aota Y, Higashi T, et al. Roentgenographic and computed tomographic findings in symptomatic lumbar foraminal stenosis. Eur Spine J 2015;24:333-8. 